\title{
|MAGERIE DANS LE BROUILLARD À LONGUE DISTANCE
}

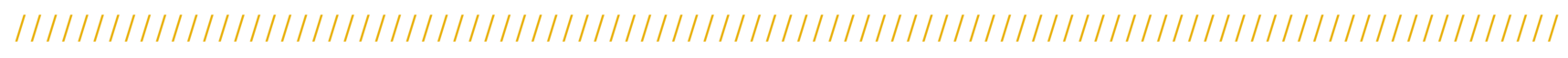

\section{Mehdi ALOUINI ${ }^{1, *}$, Fabien BRETENAKER ${ }^{2,3}$, Julien FADE ${ }^{1, *}$, Swapnesh PANIGRAH ${ }^{1, \# \#, ~ H e m a ~ R A M A C H A N D R A N ~}{ }^{3, \uparrow}$}

${ }^{1}$ Univ Rennes, CNRS, Institut FOTON, UMR 6082, F-35000 Rennes, France

${ }^{2}$ Université Paris-Saclay, CNRS, ENS Paris-Saclay, CentraleSupélec, LuMIn, Gif-sur-Yvette, France

${ }^{3}$ Light and Matter Physics Group, Raman Research Institute, Bangalore 560080, India

"Now at Aix-Marseille Univ, CNRS, Centrale Marseille, Institut Fresnel, Marseille, France

\#\#Now at CNRS, Aix-Marseille Univ, Laboratoire de Chimie Bactérienne, Institut de Microbiologie de la Méditerranée, Marseille, France

*mehdi.alouini@univ-rennes1.fr

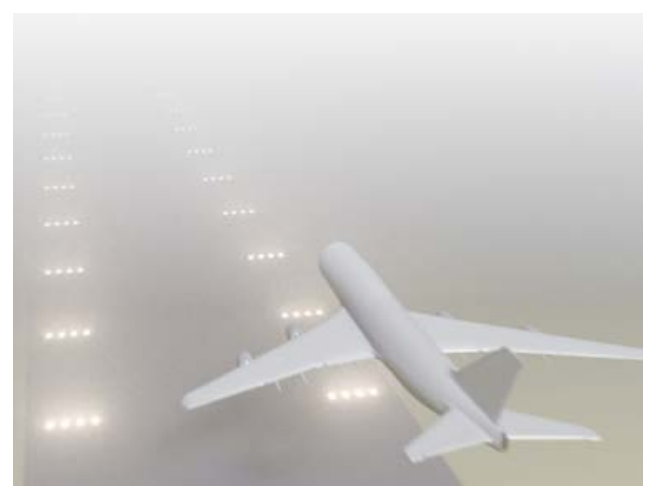

D'une rencontre en 1999 naît une aventure scientifique entre le Raman Research Institute de Bangalore (Inde) et deux laboratoires français autour de l'imagerie en milieux diffusants à longue distance pour l'imagerie en conditions de visibilité dégradées. Mêlant approches polarimétriques et modulation/démodulation temporelle des images, ces travaux ont conduit à développer plusieurs prototypes d'imageurs originaux, permettant des gains en contraste significatifs sur la visibilité de balises lumineuses en conditions de brouillard réelles.

\section{https://doi.org/10.1051/photon/202111142}

Article publié en accès libre sous les conditions définies par la licence Creative Commons Attribution License CC-BY (https://creativecommons.org/licenses/by/4.0), qui autorise sans restrictions l'utilisation, la diffusion, et la reproduction sur quelque support que ce soit, sous réserve de citation correcte de la publication originale.

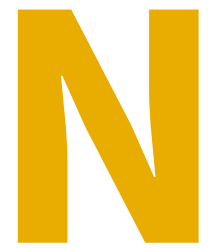

ous sommes en Inde en décembre 1999, à une rencontre scientifique franco-indienne sur les lasers et les atomes froids. Hema Ramachandran et Andal Narayanan, chercheuses au Raman Research Institute de Bangalore, viennent à la rencontre d'un des délégataires français, Fabien Bretenaker, venu de Rennes, pour échanger avec lui sur leurs travaux respectifs. En effet, il est co-auteur d'un article paru en 1996 qui décrit l'utilisation de la polarisation de la lumière pour imager des objets enfouis à l'intérieur de milieux diffusants [1]. Dans ce travail, un faisceau issu d'un laser continu polarisé linéairement passe à travers une lame demi-onde qui tourne à une vitesse angulaire $\Omega$ (voir Fig. 1a), produisant ainsi une polarisation linéaire tournante à la fréquence $2 \Omega$. Ce faisceau traverse un milieu diffusant, en l'occurrence une cuve de lait, avant d'être détecté, à travers un polariseur, et démodulé par une détection synchrone pour isoler la petite fraction de la lumière détectée qui a conservé la mémoire de la polarisation tournante. Cette technique de sélection a permis de reconstruire un objet absorbant de quelques centaines de microns de diamètre, invisible à l'œil nu au milieu de la cuve de lait (voir Fig. 1b) [1]. Il se trouve que la même idée est à la base des travaux des chercheuses indiennes [2], qui ont également utilisé une modulation de polarisation pour illuminer un milieu diffusant, mais en détectant cette fois la lumière avec une caméra CCD. Les séries temporelles d'images acquises, démodulées numériquement à la fréquence de modulation de la polarisation, leur ont permis d'obtenir des images d'objets dissimulés dans une suspension de billes de polystyrène de $0,12 \mu \mathrm{m}$ de diamètre, jouant le rôle 
de milieu à diffusion multiple de la lumière (voir Fig. 1d). De cette rencontre à Bangalore, et de ces expériences, naîtront une collaboration de plusieurs décennies, une longue amitié et des visites réciproques. Quelques années plus tard, l'idée apparaît d'appliquer ces techniques de sélection de photons pas (ou peu) diffusés pour imager des scènes à longue distance à travers le brouillard. En effet, elles pourraient constituer une solution relativement simple et économique pour la sécurisation des phases d'atterrissage d'avions par temps de brouillard, si fréquent en hiver en Inde, en disposant sur le bord de la piste des balises lumineuses modulées en polarisation (voir figure d'en-tête). L'enjeu cependant est alors de mener des expériences sur des distances kilométriques, en conditions réelles de brouillard, milieu relativement peu diffusant comparativement aux suspensions colloïdales utilisées dans les expériences de laboratoire. La collaboration s'enrichit alors de la participation de Mehdi Alouini et Julien Fade, chercheurs à l'Université de Rennes 1, et de Swapnesh Panigrahi, qui débute sa thèse sur le sujet à l'université de Rennes 1 dans le cadre d'un contrat CEFIPRA. Ces derniers disposent d'une expertise en conception de caméras polarimétriques et en traitement statistique de l'information, mais surtout ils obtiennent l'accord de la société TDF de placer un projecteur lumineux polarisé - simulant une balise de piste d'aérodrome - sur une tour de télécommunications (Fig. 2b), idéalement située à proximité du campus de Rennes... ville qui bénéficie avantageusement du brouillard indispensable pendant une partie significative de l'année ! Cette expérience sur distance kilométrique est entièrement automatisée pour être pilotable depuis l'Inde aussi bien à l'émission qu'à la réception. La détection est opérée sur un prototype de caméra polarimétrique «snapshot» optimisée pour acquérir simultanément l'image en polarisation parallèle $\left(I_{\|}\right)$et en polarisation croisée $\left(I_{\perp}\right)$ sur un même capteur CCD grâce à une architecture embarquant un prisme de Wollaston (séparateur de faisceau et polarisant) (Fig. 2a) [3]. Les enseignements tirés des campagnes expérimentales menées au cours de plusieurs hivers sont surprenants. Tout d'abord, les conclusions des expériences initiales en laboratoire ne se transposent pas dans ce contexte d'imagerie longue distance: lorsque la densité de brouillard s'accroît, et tant que de la lumière en provenance de la source est détectable à l'emplacement de la balise, celleci paraît parfaitement polarisée sur un «fond » parfaitement dépolarisé (Fig. 2b). Cette observation surprenante s'explique par la faible ouverture numérique d'un pixel individuel du détecteur, par le caractère faiblement diffusant du milieu, mais encore par la présence d'un éclairement ambiant (lumière diurne ou pollution lumineuse nocturne diffusée) fortement dépolarisé [3]. Le second enseignement crucial est que si le contraste de la balise par rapport au fond peut bel et bien être amélioré sur une image polarimétrique synthétique (obtenue par combinaison linéaire des deux canaux polarimétriques, classiquement, une image de différence $I_{\|}-I_{\perp}$ ), les coefficients de la combinaison linéaire qui maximisent le gain en contraste (Fig. 2c) dépendent fortement des conditions de visibilité de la balise, et peuvent donc varier entre deux prises de vue successives de la caméra. Cette observation, couplée à une modélisation statistique des images polarimétriques et au calcul des bornes théoriques sur les performances d'estimation, nous a permis de déterminer la représentation polarimétrique adaptative optimale (calculable en temps réel à partir du coefficient de corrélation $\rho$ des fluctuations spatiales des images), et dont les performances théoriques ont été

Figure 1. Des expériences d'imagerie en milieu diffusant par modulation de polarisation tournante, menées indépendamment en France (a) et en Inde (c), ont démontré la possibilité d'imager des objets à travers une faible épaisseur de lait (b) ou une suspension de billes de polystyrène $(d)$.

(a)

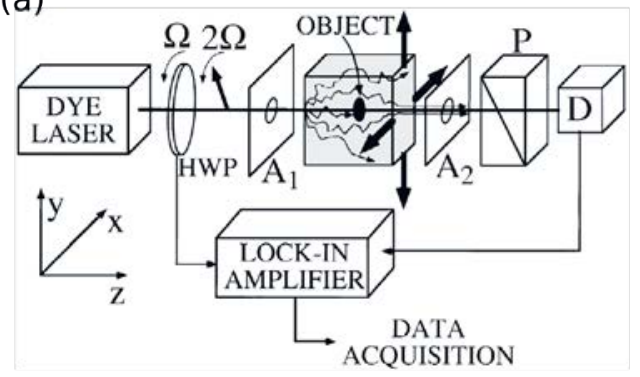

(c)

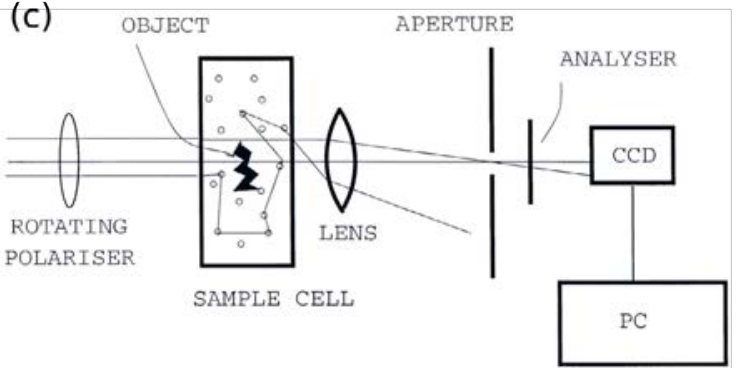

(b)

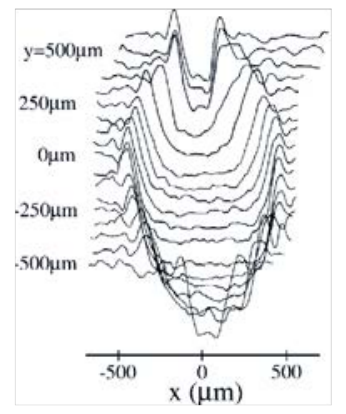

(d)

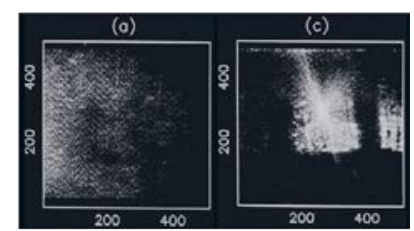


(a)

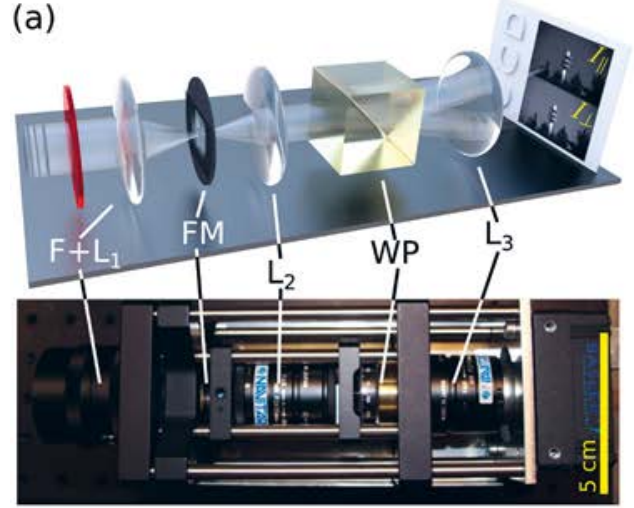

(b)

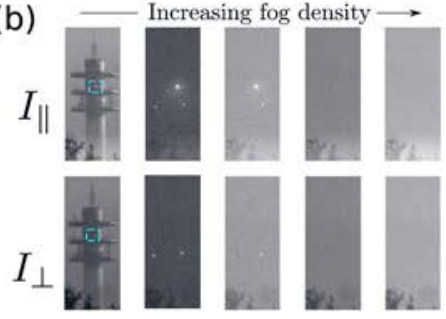

(c)

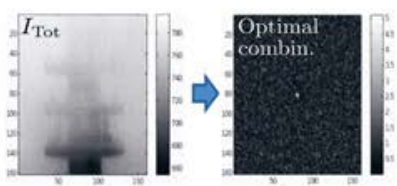

(d)

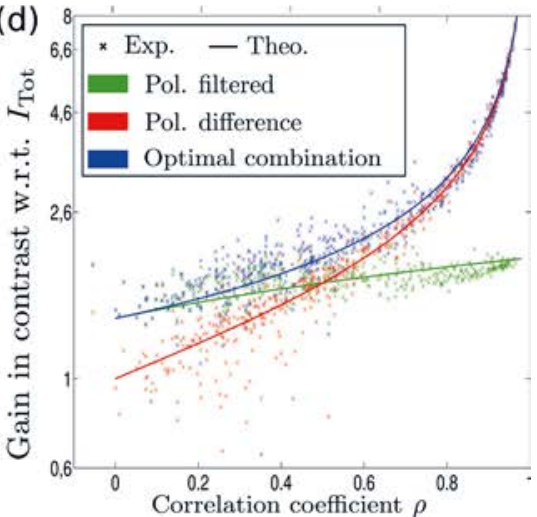

validées expérimentalement dans des conditions réelles de brouillard évoluant rapidement (Fig. 2d) [3].

En parallèle à ces travaux et au sein de la même collaboration, l'équipe indienne s'attèle de son côté à explorer le concept de démodulation temporelle de piles d'images. L'idée simple et économique consiste ici à moduler à quelques dizaines de Hertz la lumière émise (ou réfléchie) par un objet d'intérêt situé derrière un milieu diffusant, et à démoduler par post-traitement logiciel les piles d'images acquises à haute cadence sur une caméra standard. Cependant, pour répondre à l'enjeu applicatif visé, la difficulté est de parvenir à réaliser cette opération sur des images de grande taille en temps réel (à cadence vidéo pour une information
Figure 2. L'expérience d'imagerie à longue distance menée à Rennes a permis de montrer l'intérêt d'une caméra polarimétrique snapshot (a) pour augmenter significativement le contraste d'une balise lumineuse polarisée à travers un brouillard réel (b). Le gain maximal en contraste (c) est obtenu au moyen d'une combinaison optimale des deux canaux polarimétriques, dont l'expression et les performances varient en fonction du coefficient de corrélation (spatial) des fluctuations dans les images (d).

Figure 3. (a) Principe de la démodulation d'images en quadrature. (b) L'implémentation de la démodulation logicielle de piles d'images sur carte GPU a permis l'imagerie temps-réel à travers des milieux diffusants d'objets à courte distance en laboratoire (b) ou sur le terrain à distance hectométrique (c). Le gain en contraste obtenu est directement dépendant du nombre de périodes de modulation prises en compte dans l'étape de démodulation (d). destinée à un pilote), et sans devoir synchroniser émetteur (balises de piste par exemple) et récepteur (caméra embarquée sur l'aéronef). La solution proposée consiste à opérer une démodulation synchrone en quadrature à la fréquence de modulation $\Omega$, par multiplication logicielle de la pile d'images avec les deux quadratures d'un signal de référence (Fig. 3a), puis par intégration numérique sur $N$ périodes [4]. Cette approche permet d'accéder finalement à l'amplitude et à la phase de la composante de la lumière modulée à la fréquence $\Omega$, simultanément en tout pixel de l'image. La simplicité de l'algorithme autorisant aisément une parallèlisation et une implémentation sur carte graphique (GPU), nous avons démontré qu'il était possible d'accéder à un filtrage (a)
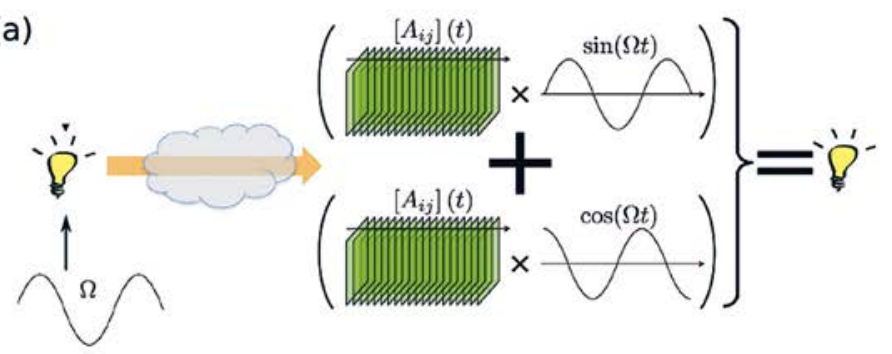

(b)

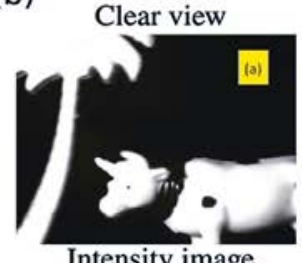

Through scattering medium

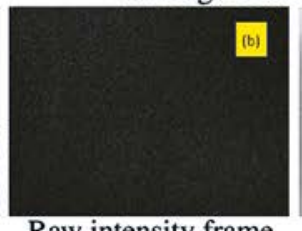

Raw intensity frame

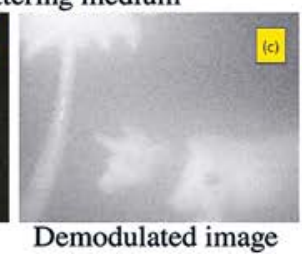

(c)
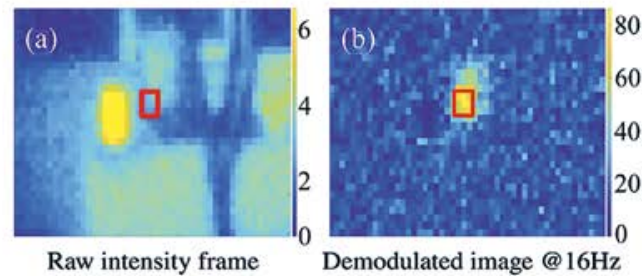

(d)

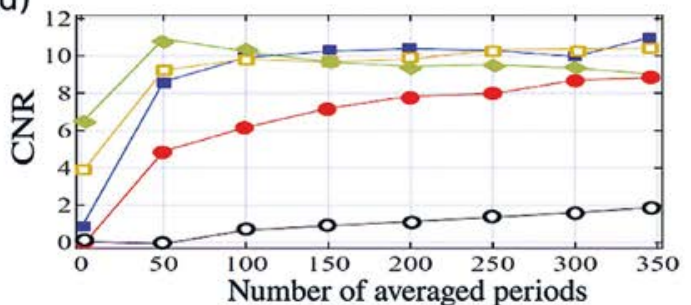


efficace des photons modulés à quelques dizaines de Hertz pour rehausser très fortement le contraste d'une scène mobile en laboratoire à cadence vidéo (Fig. 3b) [4]. Dans la lignée de ce travail, des expériences récentes menées sur le campus de l'Université Shiv Nadar dans le nord de l'Inde à travers un brouillard atmosphérique (visibilité estimée à $30 \mathrm{~m}$ ) ont permis de détecter, sans ambiguïté et à une distance de $75 \mathrm{~m}$, un objet illuminé par une source LED modulée à $16 \mathrm{~Hz}$ (voir Fig. 3c), avec un gain en contraste satisfaisant dès lors que la démodulation est opérée sur une pile d'images correspondant à quelques dizaines de périodes [4].

En «signant » les photons émis par leur polarisation ou par une modulation temporelle d'intensité, il

Les conclusions des expériences initiales en laboratoire ne se transposent pas dans ce contexte d'imagerie longue distance: lorsque la densité de brouillard s'accroît, et tant que de la lumière en provenance de la source est détectable à l'emplacement de la balise, celle-ci paraît parfaitement polarisée sur un « fond » parfaitement dépolarisé.

est donc possible de rehausser le contraste d'objets enfouis dans un fond diffus intense. Cependant, ces modalités ne permettent pas de réaliser un véritable filtrage des photons balistiques. En effet, il faudrait pour cela éliminer également la contribution de lumière diffuse provenant de la source d'intérêt (balise de piste par exemple) : l'image de celle-ci serait alors obtenue avec une résolution spatiale idéale en ne retenant que les (très rares) photons balistiques qui conservent la mémoire de leur direction de propagation d'origine. Si la technique phare dans ce domaine est l'imagerie à porte temporelle (timegated imaging), notre approche de modulation/démodulation

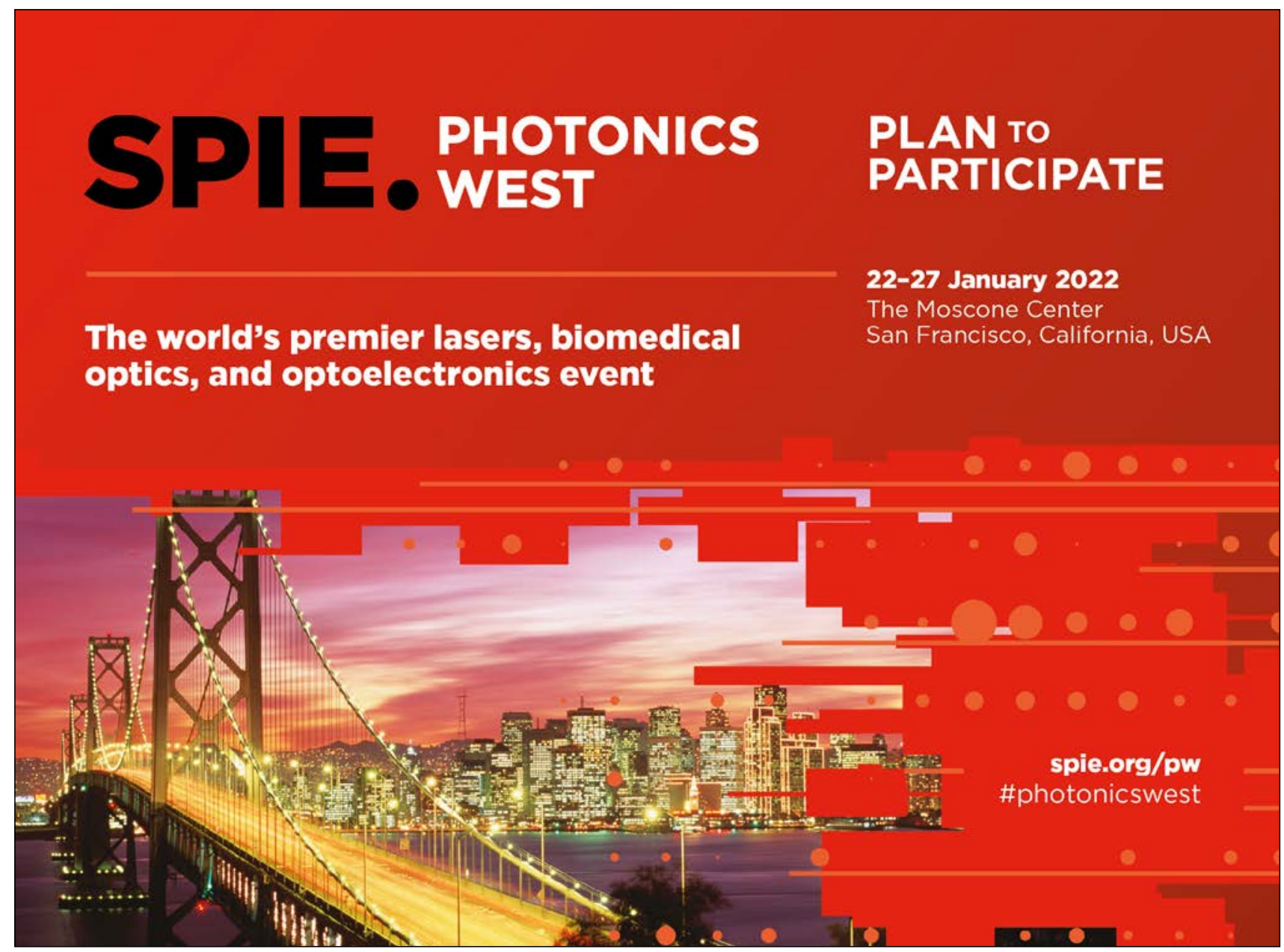


(a)
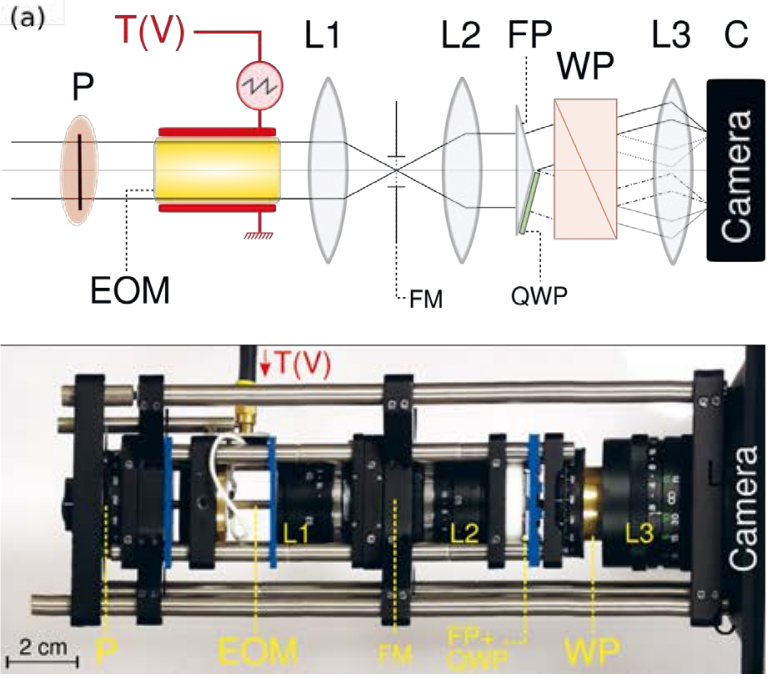

(b)
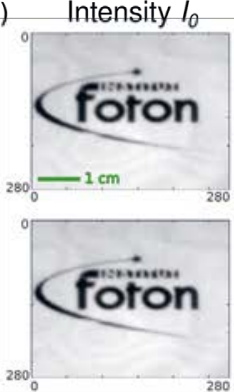

(c)

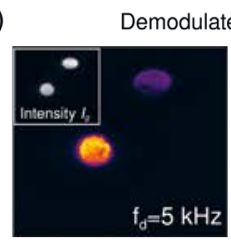

Amplitude $A$
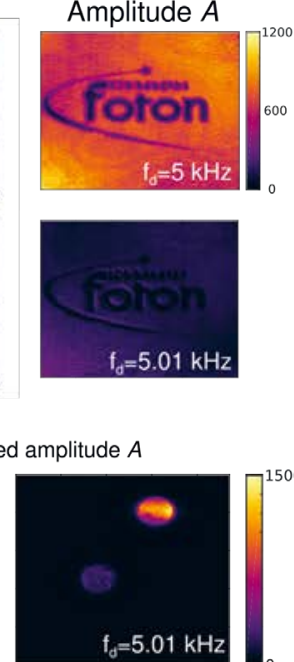

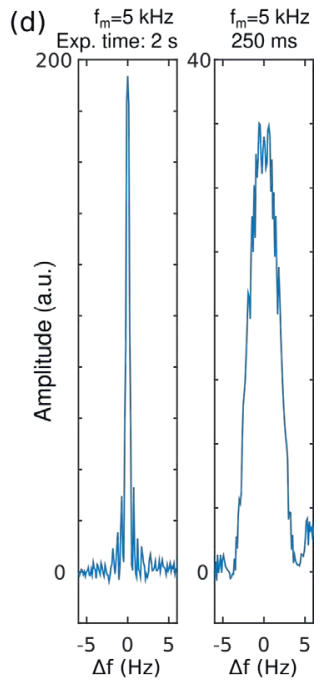

d'intensité constitue une voie d'accès duale (dans le domaine fréquentiel) possible à condition d'atteindre des fréquences de travail bien plus élevées (environ $~ 10^{8}-10^{9} \mathrm{~Hz}$ selon les propriétés du milieu diffusant). Dans cette perspective, il devenait donc incontournable d'abandonner la démodulation par voie logicielle au bénéfice d'une approche « physique ». Nous avons pour cela développé une nouvelle architecture d'imageur de démodulation plein champ, capable en théorie de fonctionner à de telles fréquences sans synchronisation de l'émetteur et du récepteur. Elle consiste en une implémentation dans le domaine polarimétrique d'une démodulation en quadrature (I/Q), dans un module tout-optique pouvant être positionné devant une caméra standard (Fig. 4a) dont le temps d'acquisition (long devant la période de modulation) offre la fonction d'intégration nécessaire à la démodulation. Au cœur du système, un cristal électro-optique est modulé par une rampe de tension à la fréquence $f_{d}$, suivi par un arrangement de composants anisotropes et séparateurs de faisceau (lame $\lambda / 4$, prisme de Fresnel et de Wollaston) pour fournir 4 images simultanées sur le détecteur en une prise de vue unique [5]. À partir des 4 images de «quadratures », il est alors possible

Figure 4. (a) Prototype de caméra de démodulation plein champ en quadrature par cristal électro-optique. (b, c) Illustration des capacités de filtrage en fréquence sur une image modulée à une fréquence unique ou contenant deux objets modulés à des fréquences proches. (d) La sélectivité fréquentielle de l'imageur est directement liée au temps d'intégration du capteur CCD.

de déduire une carte de répartition de l'intensité totale $\left(I_{0}\right)$, de l'amplitude $(A)$ et de la phase $(\varphi)$ de l'éventuelle composante modulée à la fréquence $f_{d}$ pour chaque pixel (Fig. 4b). Le premier prototype développé nous a permis de démontrer le filtrage fréquentiel sélectif de sources modulées jusqu'à environ $500 \mathrm{kHz}$ (Fig. 4c), avec une résolution fréquentielle directement donnée par l'inverse du temps d'exposition de la caméra (Fig. 4d) [5].

Le départ prématuré de Hema Ramachandran a mis un coup de frein à cette belle aventure collaborative, laissant des perspectives prometteuses à explorer. En particulier, faire fonctionner l'imageur à démodulation tout-optique à des fréquences $\mathrm{GHz}$ reste un défi technologique excitant qui devrait être relevé dans les années à venir. Il ouvrirait alors de nombreuses possibilités d'applications y compris dans le domaine de l'imagerie biomédicale.

Notre regrettée collègue et amie Hema Ramachandran nous a quittés le 24 novembre 2020 après un long et courageux combat contre la maladie.
RÉFÉRENCES

[1] O. Emile et al., Opt. Lett. 21, 1706 (1996)

[2] H. Ramachandran et al., Opt. Commun. 154, 255 (1998)

[3] J. Fade et al., Appl. Opt. 53, 3854 (2014) / S. Panigrahi et al., J. Opt. 17, 065703 (2015)

[4] S. Sudarsanam et al., Sci. Rep. 6, 25033 (2016) / S. Kumar et al., OSA Continuum 4, 1649 (2021)

[5] S. Panigrahi et al., Nat. Commun. 11, 549 (2020) 